\title{
HALL EFFECT THRUSTER DIRECT DRIVE PPUS, EXPERIMENTAL INVESTIGATION OF THE CATHODE POTENTIAL GROUNDING PROBLEM
}

\author{
Luca Ghislanzoni $^{(1)}$, Luca Benetti ${ }^{(1)}$, Tommaso Misuri ${ }^{(1)}$, Giovanni Cesaretti $^{(1)}$ Lorenzo Fontani $^{(1)}$. \\ (1) SITAEL S.p.A., via Livornese 1019, 56122 San Piero a Grado, Italy. Email: luca.ghislanzoni@sitael.com
}

\begin{abstract}
In order to maximize system mass and cost savings, a Hall Effect Thruster Direct Drive Approach shall ideally get away with the need to provide galvanic isolation for the Anode voltage. In this respect, we present some experimental data aiming at characterising the implications of a direct connection between the HET Cathode Return Potential and the Spacecraft Ground.
\end{abstract}

\section{INTRODUCTION}

Modern High Power GEO Telecom Platforms offer sufficient power capability $(20 \mathrm{~kW}$ and even more in the near future) to afford the possibility to complete, within reasonable times, the Orbit Raising phase by means of Electric Propulsion only. Considering that for such platforms a solar array and electrical power bus voltage range of $200-300 \mathrm{~V}$ represents the target of various technology developments currently being researched, significant savings could be achieved by supplying a Hall Effect Thruster (HET) Anode directly from said voltage, the so called Direct Drive approach, thus avoiding the need for the High Power Converter otherwise required for adapting the typical 100-120V bus voltage to the about $250-500 \mathrm{~V}$ Anode voltage range required by high power ( $5 \mathrm{~kW}$ and upward) Hall Effect Thrusters. At system level, such an approach would hence allow significant mass and cost savings. As an example for a $20 \mathrm{~kW}$ application, such a flight-standard converter would probably weight about $30 \mathrm{~kg}$, dissipate $>1 \mathrm{~kW}$, and cost in the range of several $100 \mathrm{~s}$ of $\mathrm{k} €$. However, maximum savings can only be achieved by renouncing to the galvanic isolation usually provided by said High Power Converter.

Concerning the subject of Hall Effect Thruster Grounding Concepts, a review of the presently available literature (see for example [1] for an introduction to the various approaches) suggests a lack of experimental data. At SITAEL we have thus decided to start an inhouse test campaign for the collection of useful experimental data on this subject.

\section{CONVENTIONAL HET GROUNDING}

On most spacecrafts utilizing electric propulsion the typical grounding approach (let us call it Floating $\boldsymbol{C R P}$ ) implements galvanic isolation between the Cathode Return Potential (CRP) and the spacecraft Electrical Power System (EPS) Ground. In practice, the otherwise floating CRP is then referenced to the EPS Ground via an RC network composed of about $200 \mathrm{nF}$ (max) in parallel with about $100 \mathrm{k} \Omega$ resistor (suitable values typically range from about $50 \mathrm{k} \Omega$ to about 200 $\mathrm{k} \Omega$ ). Any beam stray current, $\boldsymbol{I}_{\boldsymbol{B} S \boldsymbol{C}}$, which may "leak" to the EPS Ground, through a plurality of complex diffusion and interaction mechanisms involving some of the spacecraft conductive surfaces exposed to the plasma environment, would then result in a voltage drop across R. Such voltage drop would thus move the CRP in the direction of reducing $\boldsymbol{I}_{\boldsymbol{B} S \boldsymbol{C} C}$, until a point of equilibrium is finally achieved. Typically, at equilibrium, the CRP can reach few tens of volts negative with respect to the EPS Ground, but also the case with CRP few volts positive cannot be entirely ruled out (as shown by measurements performed on-

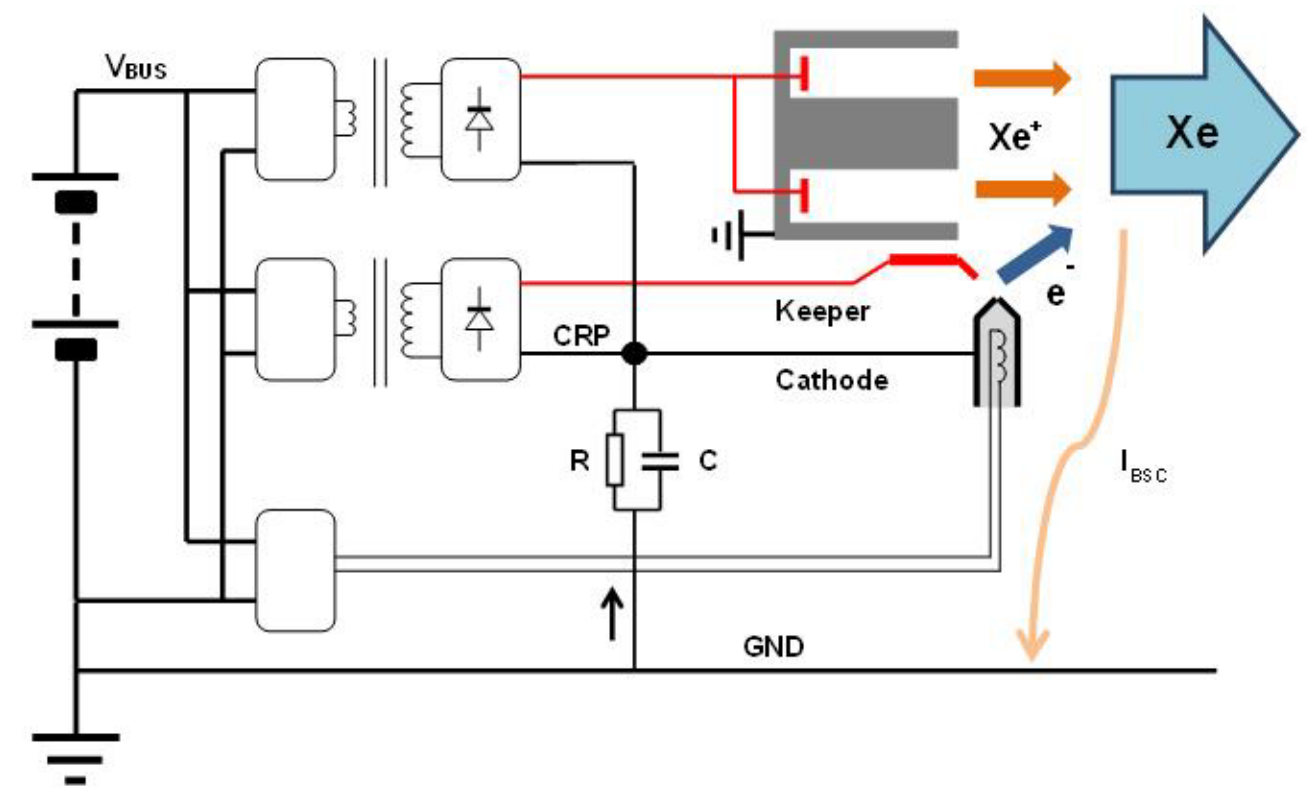


board during the SMART-1 mission [2]). In fact, given a certain value of R, the actual value of $\boldsymbol{I}_{\boldsymbol{B S C}}$ will depend on the value of relevant environmental variables:

- plasma environment

- orbit altitude

- $\quad$ sunlight or eclipse

- solar activity

- season

It seems obvious that a test bed environment might significantly differ from the actual orbital environment, at least for what concerns:

- the level of vacuum achievable

- the much larger collecting surface for $\boldsymbol{I}_{\boldsymbol{B S C}}$ (the internal walls of the chamber, which is grounded), with respect to the exposed conductive surfaces of a spacecraft.

By definition, such a Floating $\boldsymbol{C R P}$ approach requires the full anode power to undergo conversion (i.e. dc $\rightarrow$ ac $\rightarrow$ transformer $\rightarrow$ rectifier $\rightarrow$ anode voltage) in order to provide galvanic isolation, and hence incurring in the mass and cost penalties recalled above.

\section{THE GROUNDED CRP APPROACH}

Ultimately, a Direct Drive application would imply a Grounded $\boldsymbol{C R P}$ approach, consisting in forcing the CRP potential to the spacecraft Ground potential. In such a condition the current flowing between the CRP and the Test Bed Ground, coincident with $\boldsymbol{I}_{\boldsymbol{B S C}}$, will need to be monitored in order to study its dependence from the relevant environmental variables. The functional diagram below helps in understanding the Grounded $\boldsymbol{C R P}$ experimental setup.

The Grounded $\boldsymbol{C R P}$ configuration, corresponding to what a typical Direct Drive application would require, is obtained when SW1 is closed. In such a situation one shall consider the possibility that after the cathode has reached its nominal operating temperature, and thus thermally emitted electrons are already present, and the flow of Xenon is started, the application of the discharge-igniting pulse at the cathode Keeper electrode might instead strike a discharge to some ground referenced conductive parts others than the intended Cathode electrode. Such potential catastrophic impossibility to ignite the Cathode discharge can be prevented by adopting galvanic isolation (with respect to the EPS Ground) of the Keeper voltage, while also adding diode $\boldsymbol{D}$ as to reverse block any current discharge path not striking the Cathode. In other words, by design (because of the galvanic isolation) the igniting discharge current is in this way forced to flow between the positive and return outlets of the Keeper supply. Hence, connecting said Keeper return directly to the Cathode, while decoupling it from the Main Ground by means of diode $\boldsymbol{D}$, will ensure that any Keeper current will automatically be forced to return through the Cathode. Once the Anode discharge is also ignited, diode $\boldsymbol{D}$ will then allow the bulk of $\boldsymbol{I}_{\boldsymbol{A} N}$ to flow through. For diagnostic and characterization purposes, a current sensor $(\boldsymbol{A})$ will monitor the value of $\boldsymbol{I}_{\boldsymbol{B S C} \text {. }}$

With both SW1 and SW2 closed it will then be possible to verify under what conditions the risk of not being able to correctly ignite the cathode discharge might become a concern. The following $I_{B S C}\left(V_{C R P}-V_{G N D}\right)$ characterization was performed:

- $\quad \boldsymbol{S W 1} \& \boldsymbol{S W 2}$ open: while the thruster is firing, the output voltage of a two quadrant (i.e. capable of both sourcing and sinking current) laboratory supply ( $\boldsymbol{V}$ in the figure above), can be adjusted from few $10 \mathrm{~s}$ of Volts negative to few $10 \mathrm{~s}$ of Volts positive, and plots of $\boldsymbol{I}_{B S C}\left(\boldsymbol{V}_{C R P}-V_{G N D}\right)$ obtained.

- $\quad \boldsymbol{S W 1} \& \boldsymbol{S W 2}$ closed: $\boldsymbol{V}$ is then set to 0V and SW1 closed, as to obtain a Grounded $\boldsymbol{C R P}$ configuration. Once the thruster is stably firing, SW2 can also be closed in order to avoid unnecessary power dissipation through diode $\boldsymbol{D}$. The measured values of $\boldsymbol{I}_{\boldsymbol{B S C}}$ are then recorded while sweeping the thruster through various operating points.

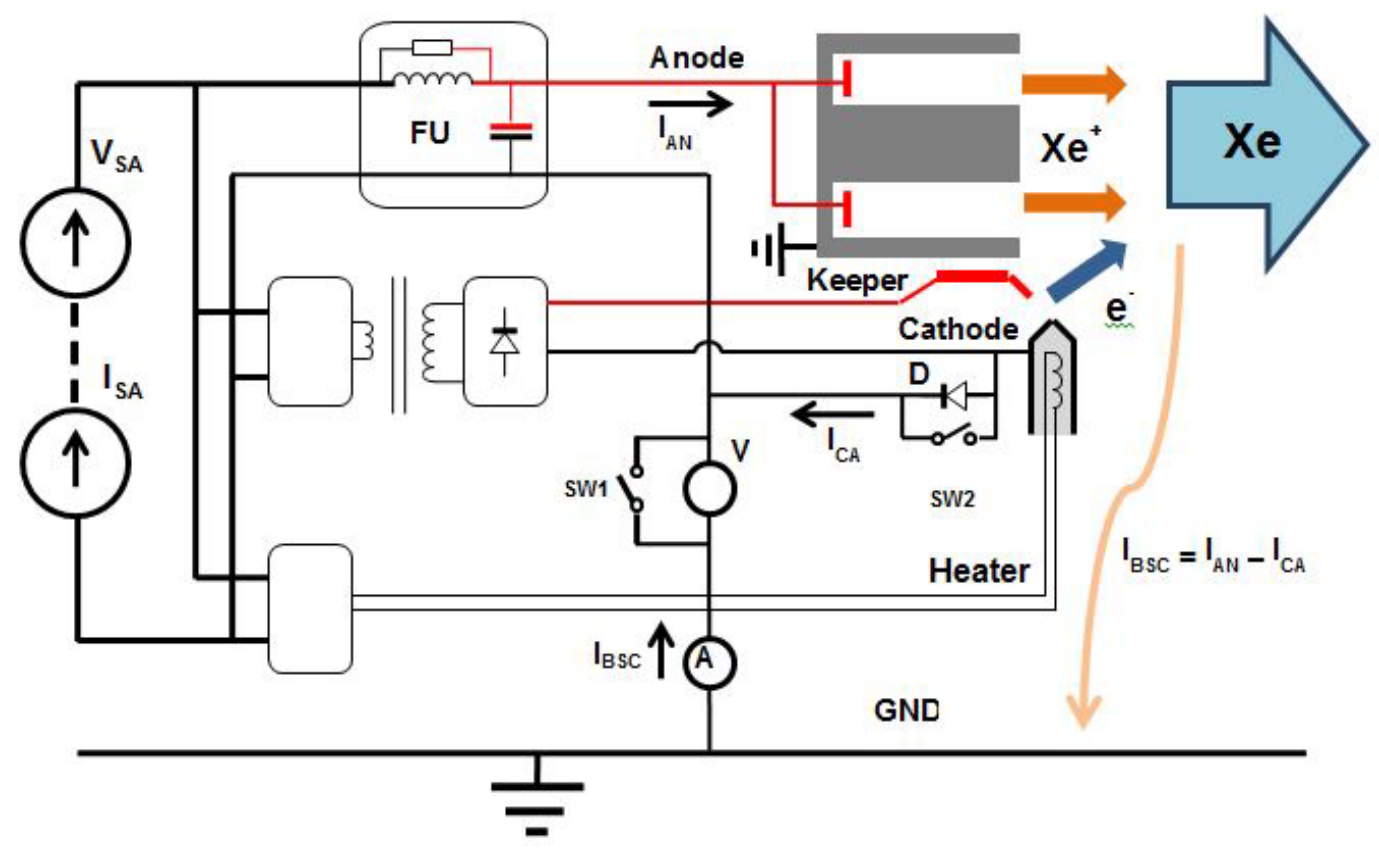


Although said $\boldsymbol{I}_{\boldsymbol{B S C}}\left(\boldsymbol{V}_{\boldsymbol{C R P}}-\boldsymbol{V}_{\boldsymbol{G N D}}\right)$ characterization, performed in a vacuum chamber test setup, might not be fully representative of what may be observed in an actual orbital environment, we believe that its comparison with similar experimental data that will be later collected during the $\mu$ HET In Orbit Validation Mission of SITAEL's low power HT-100 thruster may nevertheless provide useful indications. Such set of experimental data will aid in assessing the actual differences between the two environments, possibly suggesting with respect to which phenomenon one can be considered worst case with respect to the other. Furthermore, such set of experimental data could later be used for helping in the validation of models developed for the prediction of such effects.

In this respect a recent paper from Katz et al. [3] suggests that:

"The most important parameters that go into predicting cathode common voltages are solar array electron collection and the thruster body I-V curve. Both of these can be measured in laboratory tests. This data combined with a Hall thruster plume model such as HALLPLUME2D and interactions codes, e.g. NASPAS can be used to choose the value of the resistor that both isolate the spacecraft chassis from thruster power system noise and reduce cathode common voltages to prevent undue sputter damage."

By following the suggested methodology it appears therefore possible, starting from laboratory measurements, to predict the above mentioned $I_{B S C}\left(V_{C R P}-V_{G N D}\right)$ Volt - Ampere characteristics.

\section{TEST SETUP}

We have thus proceeded with a first measurement of said $\boldsymbol{I}_{\boldsymbol{B S C}}\left(\boldsymbol{V}_{\boldsymbol{C R P}}-\boldsymbol{V}_{\boldsymbol{G N D}}\right)$ Volt - Ampere characteristics for the following example:

- $\quad$ HET was SITAEL's HT 5K operated at $2.5 \mathrm{~kW}$.

- The firing chamber was SITAEL's IV4 facility.

- The voltage between CRP and GND was forced by means of a two-quadrants power supply.

The IV4 steel chamber has a diameter of $2 \mathrm{~m}$ and a total volume of about $8 \mathrm{~m}^{3}$. The facility is equipped with a series of cryogenic heads, a cryogenic pump and two turbomolecular pumps. IV4 can reach an ultimate vacuum level in the $10^{-8}$ mbar range.

This vacuum chamber is equipped with a complete array of diagnostics, including plasma probes, as well as nonintrusive capabilities such as infrared thermography, ultra-high speed imaging and spectroscopy.

In order to damp the plasma beam energy and to reduce the contamination of the thruster due to back-sputtering from the facility's walls during long duration tests, a biconical beam target lined with high purity graphite, has been designed and accommodated in front of the thruster inside the opening cap of the facility.

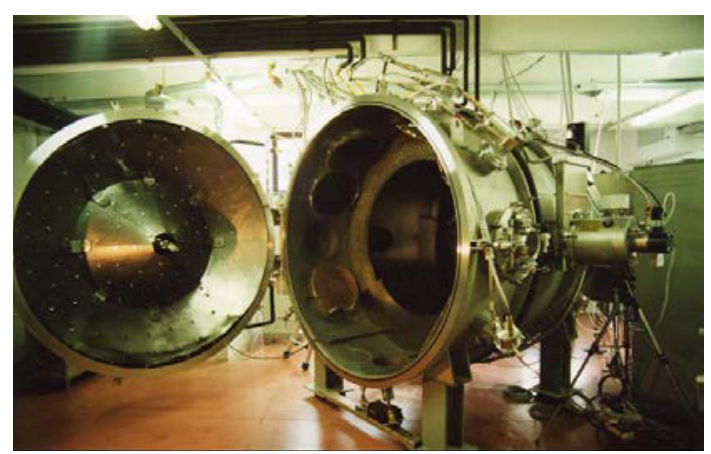

SITAEL's IV4 facility (open during servicing).

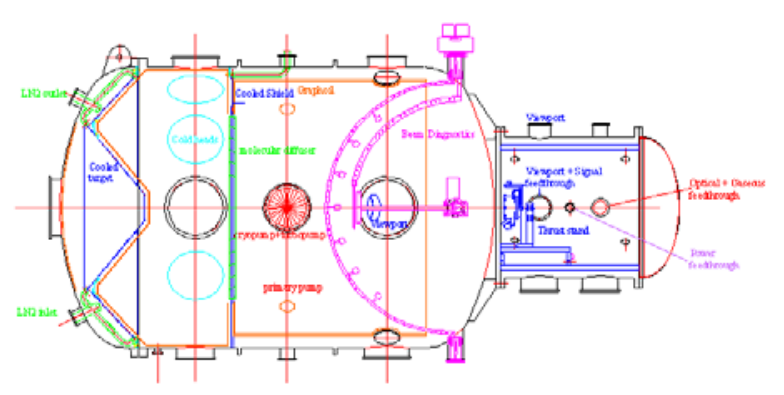

IV4 facility schematics.

\section{MEASUREMENT RESULTS}

The measured $\boldsymbol{I}_{\boldsymbol{B S C}}\left(\boldsymbol{V}_{\boldsymbol{C R P}}-\boldsymbol{V}_{\boldsymbol{G N D}}\right)$ Volt - Ampere characteristic is illustrated in the following graph:

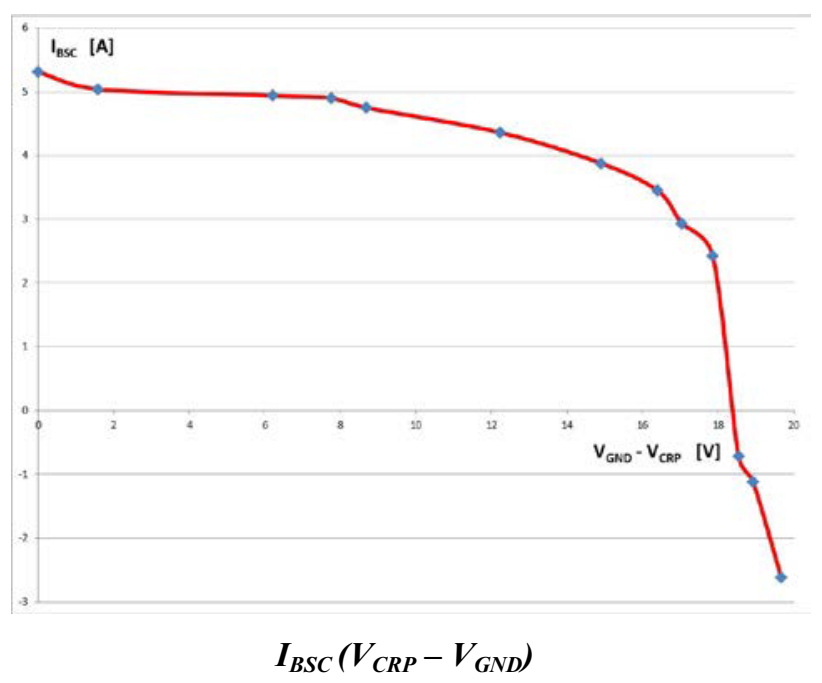

about which we can make the following observations:

- $\boldsymbol{I}_{\boldsymbol{B} S \boldsymbol{C}}$ changes sign at about the same $\boldsymbol{V}_{\boldsymbol{C R} \boldsymbol{P}}$ value which the cathode would reach at equilibrium with the conventional Floating $\boldsymbol{C R P}$ approach.

- Slowly varying the voltage of the two quadrants supply (i.e. slowly varying $\boldsymbol{V}_{\boldsymbol{G N D}}-\boldsymbol{V}_{\boldsymbol{C R P}}$ ) around said equilibrium voltage results in a large variation of $\boldsymbol{I}_{B S C}$.

We are not aware of any such $\boldsymbol{I}_{\boldsymbol{B S C}}\left(\boldsymbol{V}_{\boldsymbol{G N D}}-\boldsymbol{V}_{\boldsymbol{C R P}}\right)$ measurements performed on flying spacecrafts. 


\section{CRP BIAS POWER SUPPLY}

At first sight it may appear that a Direct Drive approach would allow a considerable simplification of the Power Processing Unit, thanks to the removal of the Anode Voltage Supply. However, if a direct connection to GND of the Cathode Potential may result in a large $\boldsymbol{I}_{\boldsymbol{B S C}}$ current also when in an actual orbital environment, then galvanic isolation of the anode voltage would anyway appear to become a practical necessity. Thus falling back again to the need for a massive and costly Anode Voltage Supply. Although a laboratory firing chamber environment might significantly differ from the actual environment experienced in orbit, we nevertheless believe that flying, on a large GEO Telecom platform, a Direct Drive HET propulsion system with its CRP directly connected to GND might expose the spacecraft to the risk of potential damages resulting from a too large $\boldsymbol{I}_{B S C}$ current.

Of course, the very same test setup illustrated above suggests an interesting alternative: insert a series power supply between the CRP and GND (see simplified schematics at the bottom of this page). Said "CRP Bias Supply" would then typically need to handle solely about $1 / 20^{\text {th }}$ to $1 / 10^{\text {th }}$ the power of the otherwise required Anode Supply, considerably reducing cost, mass, volume, and burden on the spacecraft thermal control system.

The negative output voltage of said CRP Bias Supply could then be controlled in order to achieve the condition $\boldsymbol{I}_{\boldsymbol{B S C}}=\boldsymbol{0}$. Such a feedback loop would need to measure $\boldsymbol{I}_{\boldsymbol{B S C}}$ as the difference between the positive anode current and the cathode return current. A differential current measurement of this kind can optimally be obtained by means of a Hall Effect Current Sensor coupled to both currents by means of two sensing windings in oppositions.

Because the adjustments to the output voltage of said CRP Bias Supply would need to follow only the very slow variations of $\boldsymbol{I}_{\boldsymbol{B S C}}$ (as determined by the slowly varying orbital environment), the actual controller for said feedback loop could then be implemented by the FPGA hosting also the overall PPU Sequencer and Controller.

\section{CONCLUSIONS}

A Direct Drive approach to the supply of the anode voltage for a Hall Effect Thruster would reap major benefits (mass, cost, dissipation) if it were possible to directly connect the Cathode Potential to the spacecraft's Ground. However, this may result in damaging $\boldsymbol{I}_{\boldsymbol{B} \boldsymbol{S} \boldsymbol{C}}$ values. It has been shown how an interesting solution might consist in introducing, between CRP and GND, a CRP Bias Supply controlled by means of a feedback loop aimed at minimizing $\boldsymbol{I}_{\boldsymbol{B S C} \boldsymbol{C}}$.

Said CRP Bias Supply would then typically need to process only about $1 / 20^{\text {th }}$ to $1 / 10^{\text {th }}$ of the actual HET anode power, and thus resulting in significant savings.

\section{REFERENCES}

1. Gollor, M. (2011). "Accommodation Aspects of Electric Propulsion with Power Processing Units and the Spacecraft" 9th Annual International Energy Conversion Engineering Conference, AIAA 2011-5651.

2. Koppel, C. R., Marchandise, F., Estublier, D. \& Jolivet, L. (2004). The SMART-1 Electric Propulsion Subsystem In Flight Experience. AIAA-2004-3435.

3. Ira Katz et al. "Effect of Solar Array Plume Interactions on Hall Thruster Common Potentials". Spacecraft Charging Technology Conference 04 08 April 2016, ESA/ESTEC.

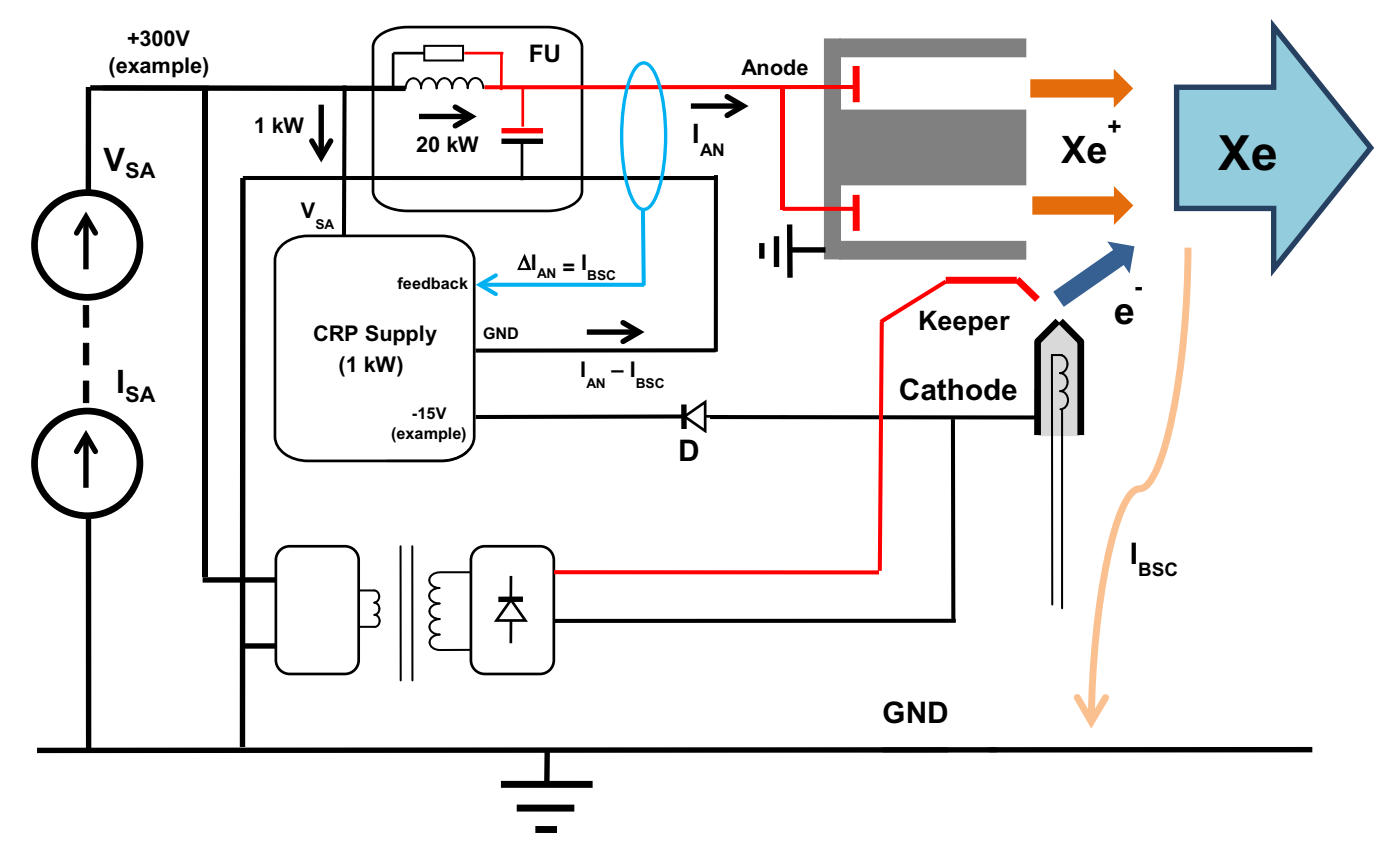

